\title{
NEW FORMS OF HIGH ENERGY DENSITY MATTER
}

\author{
LARRY MCLERRAN \\ Nuclear Theory Group, Brookhaven National Laboratory, Upton, NY 11793
}

In this talk, I discuss the scientific issues being addressed in ultrarelativistic heavy ion collisions. I also discuss some of the recent results from RHIC at Brookhaven National Laboratory which give some experimental insight into these issues.

\section{High Density Matter}

\subsection{The Goals of RHIC}

The goal of nuclear physics has traditionally been to study matter at the highest possible densities. This has been done in the past by studying the atomic nucleus, where energy densities become of the order of

$$
\epsilon \sim .15 \mathrm{GeV} / \mathrm{Fm}^{3}
$$

High energy nuclear physics has extended this study to energy densities several orders of magnitude higher. This extension includes the study of matter inside ordinary strongly interacting particles, such as the proton and the neutron, and producing new forms of matter at much higher energy densities in high energy collisions of nuclei with nuclei, and various other probes.

RHIC is a multi-purpose machine which can address at least two issues related to the properties of matter at very high energy density. These are:

- The production of matter at energy densities one to two order of magnitude higher than that of nuclear matter and the study of its properties.

This matter is at such high densities that it is only simply described in terms of quarks and gluons and is generically referred to as the Quark Gluon Plasma. The study of this matter may allow us to better understand the origin of the masses of ordinary particles such as nucleons, and of the confinement of quarks and gluons into hadrons. The Quark Gluon Plasma will be described below. 
- The study of the matter which controls high energy strong interactions.

This matter is believed to be universal (independent of the hadron), and exists over sizes enormous compared to the typical microphysics size scales important for high energy strong interactions. (The microphysics time scale here is roughly the size scale here is about $1 \mathrm{Fm}$ and the microphysics time scale is the time it takes light to fly $1 \mathrm{Fm}, t \sim 10^{-23} \mathrm{sec}$.) It is called a Color Glass Condensate because it is composed of colored particles, gluons, evolves on time scales long compared to microphysics time scales and therefore has properties similar to glasses, and a condensate since the phase space density of gluons is very high. The study of this matter may allow us to better understand the typical features of strong interactions when they are truly strong, a problem which has eluded a basic understanding since strong interactions were first discovered. The Color Glass Condensate will be described below.

\subsection{The Quark Gluon Plasma}

This section describes what is the Quark Gluon Plasma, why it is important for astrophysics and cosmology, and why it provides a laboratory in which one can study the origin of mass and of confinement. [1]

\subsubsection{What is the Quark Gluon Plasma?}

Matter at low energy densites is composed of electrons, protons and neutrons. If we heat the system, we might produce thermal excitations which include light mass strongly interacting particles such as the pion. Inside the protons, neutrons and other strongly interacting particles are quarks and gluons. If we make the matter have high enough energy density, the protons, nucleons and other particles overlap and get squeezed so tightly that their constituents are free to roam the system without being cofined inside hadrons. At this density, there is deconfinement and the system is called a Quark Gluon Plasma. This is shown in Fig. 1

As the energy density gets to be very large, the interactions between the quarks and gluons become weak. This is a consequence of the asymptotic freedom of strong interactions: At short distances the strong interactions become weak. 


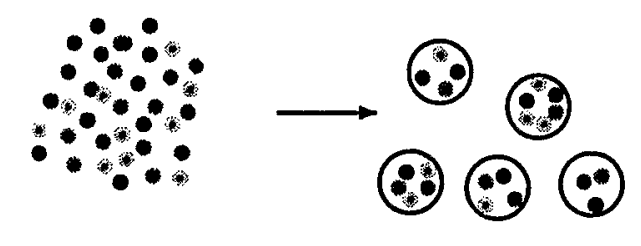

\section{Quark-Gluon Plasma $\quad \longrightarrow \quad$ Hadron Gas}

Figure 1: As the energy density is decreased, the Quark Gluon Plasma condens into a low density gas of hadrons.

\subsubsection{The Quark Gluon Plasma in Cosmology and Astrophysics}

During the big bang, matter presumably existed at temperatures as high as the Planck scale. This matter expanded and cooled until present densities. During this expansion it went through a number of phase transitions, and these transitions had implications for the production of matter as we know it. The various stages in the early big bang are shown in Fig. 2. (We measure temperature in energy units. In these units, the energy of a typical thermal fluctuation is the temperature.)

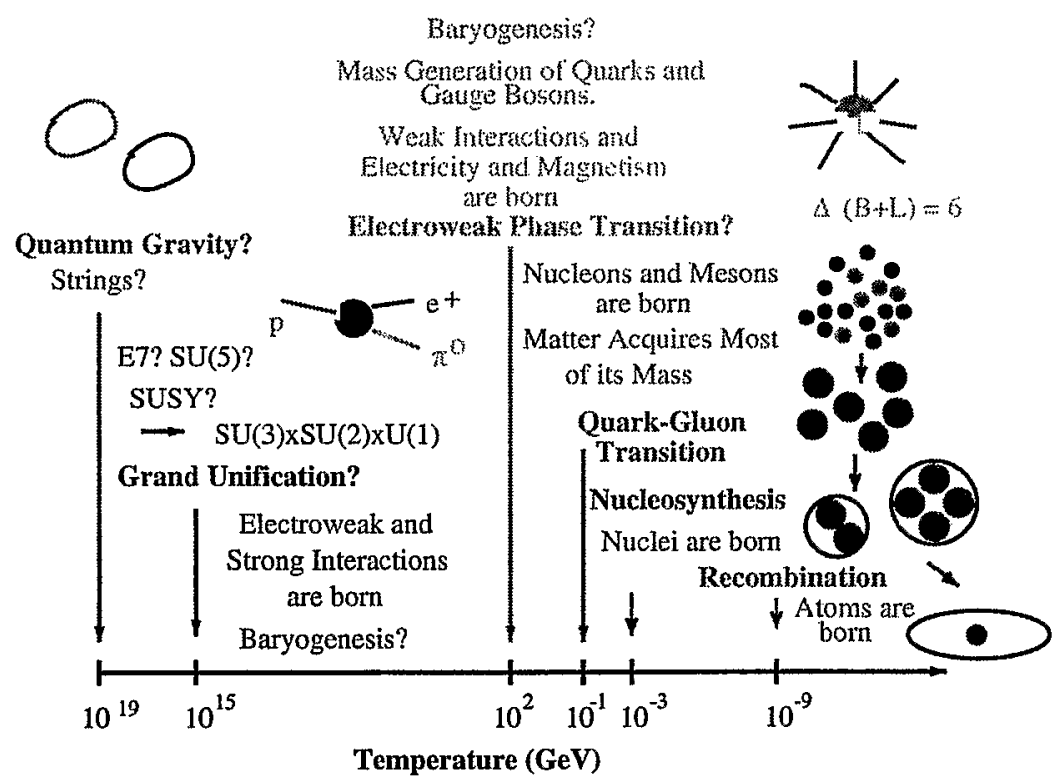

Figure 2: The early history of the universe. 
The lowest temperature shown in the plot is about $1 \mathrm{eV}$, which is the temperature at which atoms form. At this temperature, the pressure drops dramatically and stucture formation begins. At the earliest times, little is known. It could be that at temperatures of the order of the Planck scale that the initial conditions for the big bang are themselves determined by quantum gravity. Perhaps this is where the interaction strengths of all particles are determined. At a temperature of the order of $T \sim 10^{15} \mathrm{GeV}$, perhaps the strong interactions and electroweak interactions are born from a phase transition which splits the strong interactions from those of electroweak. Perhaps the matter asymmetry of the universe is born here? (The matter or baryon number asyymmetry is simply that there is much more matter than anti-matter.)

Of course the physics at the earliest times and highest temperatures is extremely speculative. At lower temperatures, we think we understand the fundamental theories of particle interactions. At the scale of temperatures corresponding to the electroweak boson, $T \sim 100 \mathrm{GeV}$ the electromagnetic and weak interactions are born from the electroweak, which until this scale were one unified interaction. Small masses of order of tens of MeV's are generated for the light quarks, and the masses of the heavy W, Z and Higgs bosons are generated. Perhaps also the baryon asymmetry is generated by coherent interactions of the $\mathrm{W}$ and $\mathrm{Z}$ fields. At a temperature of the order of $T \sim 100 \mathrm{MeV}$, quarks and gluons condense into hadrons, and quarks become permanently confined. Here $97 \%$ of the mass of nucleons is created. At a temperature of the order of $1 \mathrm{MeV}$, nucleons bind into light nuclei. The abundances of hydrogen, deuterium, helium and lithium have been predicted and agree with observation. At a temperature of order of $1 \mathrm{eV}$, atoms are made, and structure formation begins.

The Quark Gluon Plasma transition occurs at temperatures of order of hundreds of $\mathrm{MeV}$. This is where mass generation and confinement occur. The QCD phase transition is the only one of all the cosmological phase transitions which can be studied experimentally. This is because the energy scales associated with the phase transitions at higher temperatures are too large for one to imagine making matter in bulk in any foreseeable laboratory environment. One might say that we can understand these transitions without laboratory experiment since after all the fundamental theory is understood. Unfortunately, there will likely be unexpected dynamical effects, which are difficult to predict from first principles knowledge of the underlying theory alone. This transition is incredibly strong, where the change in the energy per particle associated with the transition is of the order of the rest masses of 
particles. We have no experience with such strong transitions.

In astrophysics, quark-gluon matter can occur in neutron stars, supernovae and gamma ray bursters. In Fig. 3, we show the current theoretical conception of a neutron star. Neutron stars have densities near the surface close to the densities in atomic nuclei, and towards the core densities of about an order of magnitude greater. In the core, the density corresponds roughly to the energy density inside a single proton and it is reasonable to expect that this matter is quark matter. Recent computations suggest that this matter may have very novel properties and have a variety of superconducting phases associated with colored and spinning Cooper pairs.

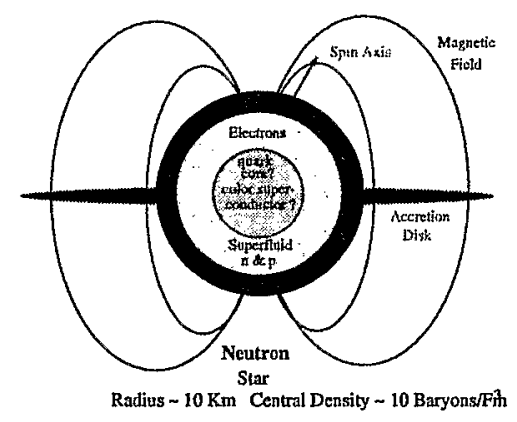

Figure 3: The interior of a neutron star.

Gamma ray bursters produce high energy photons, $E \sim 1 \mathrm{MeV}$, and these photons carry an total amount of energy which may be as large as thatof the typical gravitational binding energy of a neutron star. This is of the order of $100^{\prime} s$ of $\mathrm{MeV}^{\prime} s$ per nucleon times the number of nucleons in the star. A popular model of gamma ray bursts is the collision of two neutron stars. Because the gravitational binding energy is large, this is in fact the ultimate relativistic heavy ion collision. The end product of this collision is a hypernova of dense matter.

\subsubsection{The Quark Gluon Plasma and Fundamental Physics Issues}

A hypothetical phase diagram for QCD is shown in Fig. 4 The vertical axis is temperature, and the horizontal is a measure of the matter or baryon number density, the baryon number chemical potential. The solid lines indicate a first order phase transition, and the dashed line a rapid cross over. It is not known whether or not the region marked cross over is or is not a true first order phase transition. There are analytic arguments for the properties of matter at high 
density, but numerical computation are of insuffucient resolution. At high temperature and fixed baryon number density, there are both analytic arguments and numerical computations of good quality. At high density and fixed temperature, one goes into a superconducting phase, perhaps multiple phases of superconducting quark matter. At high temperature and fixed baryon number density, the degrees of freedom are those of a Quark Gluon Plasma.

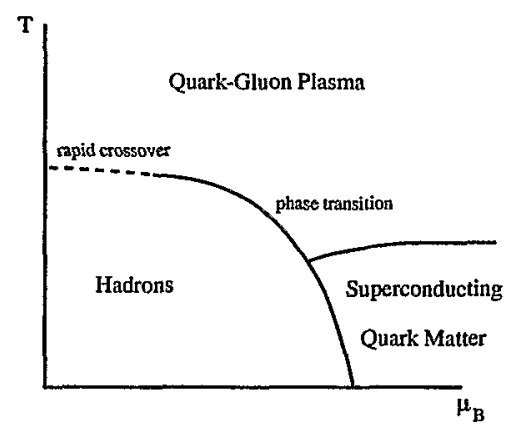

Figure 4: A phase diagram for QCD collisions.

Along the temperature axis at zero baryon number density, one can do numerical Monte-Carlo simulations of QCD to measure the properties of the Quark Gluon Plasma. One can measure the number of degrees of freedom as a function of temperature since they are $N_{\text {dof }} \sim \epsilon / T^{4}$. (Recall that at temperatures high compared to that of particle masses, the distribution of particles is that of a black body. They change very rapidly, perhaps even discontiuously, at a temperature of about $T_{c} \sim 160-190 \mathrm{MeV}$ between a number typical of a gas with pion degrees of freedom and those of a qaurk gluon plasma. The number of degrees of freedom for a Quark Gluon Plasma is about 50 (corresponding to eight gluons with 2 spins each and 3 generations of quarks and antiquarks with 2 spins each and three colors. $N_{\text {dof }} \sim 2 \times 8+3 \times 3 \times 2 \times 2$ ), whereas that for a pion gas is 3 corresponding to the three possible charge states of a spinless pion). This is shown in Fig. 5

The generation of mass in strong interactions is believed to be associated with the breaking of a symmetry. This symmetry if unbroken would allow for only very small mass for nucleons and would have a large mass for the pion, the lightest of all mesons. The breaking of this symmetry is analogous to the breakdown of rotational invariance in a magnetized solid. In nature, chiral symmetry is broken, nucleons have large masses and the pion has a very small mass. (In the analogy with magnetism, the pion corresponds to a spin 


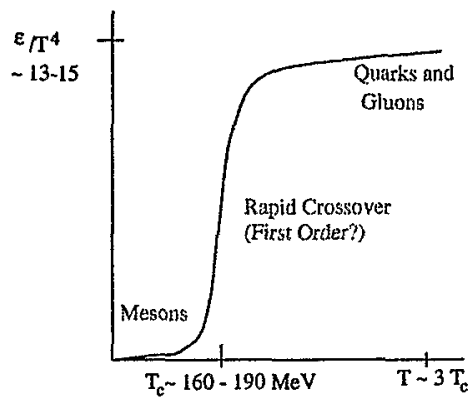

Figure 5: The number of degrees of freedom of matter as a function of temperature.

wave.) The nucleon mass is proportional is an order parameter denoted by $<\bar{\Psi} \Psi>$ which is roughly proportional to the nucleon mass. (This is analogous to the magnetization.) In Fig. 6, this is plotted against $T$. The nucleon mass is indeed small at high temperature, and it would appear that the QCD transition is responsible for the generation of about $97 \%$ of the nucleon mass. The transition occurs at the same temperature where the number of degrees of freedom change between that of a pion gas and a Quark Gluon Plasma.

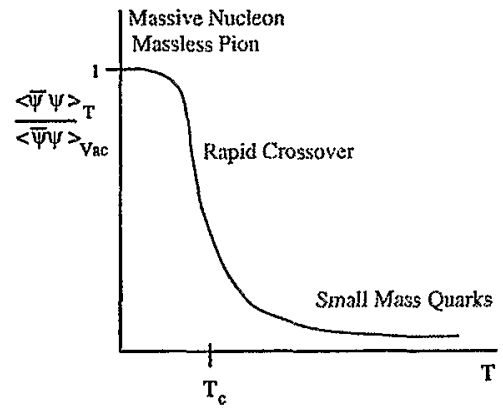

Figure 6: A nucleon mass as a function of temperature.

A plot of the potential between two heavy quarks is shown in Fig. 7. At high temperature, the potential tends to a constant and quarks are not confined. At low temperature, the potential is linear and they are confined, since isolated quarks would have infinite energy. The transition occurs at the Quark Gluon Plasma transition temperature.

The generation of nucleon masses and confinement has many theoretical explanations, but few experimental tests. One of the beauties of high tempera- 


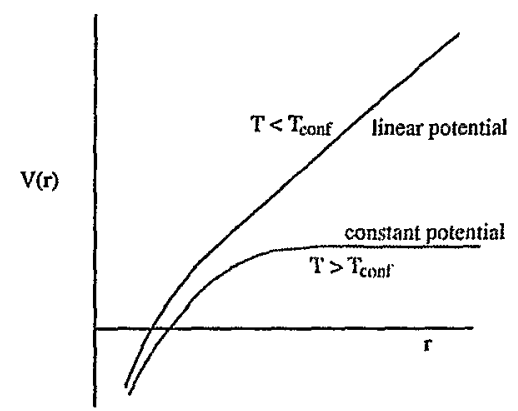

Figure 7: The quark potential a function of temperature.

ture QCD is that it allows one to turn on and off the effects of confinement and mass generation by tuning the temperature, and this should allow for deeper understanding.

\subsection{The Color Glass Condensate}

This section describes what is the Color Glass Condensate, and why it is important for our understanding of basic properties of strong interactions. We argue that the Color Glass Condensate is a universal form of matter which controls the high energy limit of all strong interaction processes and is the part of the hadron wavefunction important at such energies. Since the Color Glass Condensate is universal and controls the high energy limit of all strong interactions, it is of fundamental importance.[2]

\subsubsection{What is the Color Glass Condensate?}

A very high energy hadron has contributions to its wavefunction from gluons, quarks and anti-quarks with energies up to that of the hadron and all the way down to energies of the order of the scale of light mass hadron masses, $E \sim 200 \mathrm{MeV}$. A convenient variable in which to think about these quark degrees of freedom is the typical energy of a constituent scaled by that of the hadron,

$$
x=E_{\text {constituent }} / E_{\text {hadron }}
$$

Clearly the higher the energy of the hadron we consider, the lower is the minimum $x$ of a constituent. Sometimes it is also useful to consider the rapidity of a constituent which is $y \sim \ln (1 / x)$ 
The density of small $\mathrm{x}$ partons is

$$
\frac{d N}{d y}=x G\left(x, Q^{2}\right)
$$

The scale $Q^{2}$ appears because the number of constituents one measures depends (weakly) upon the resolution scale of the probe with which one measures. (Resolution scales are measured in units of the inverse momentum of the probe, which is usually taken to be a virtual photon.) A plot of $x G\left(x, Q^{2}\right)$ for gluons at various $x$ and $Q^{2}$ measured at the HERA accelerator in protons is shown in Fig. 8.

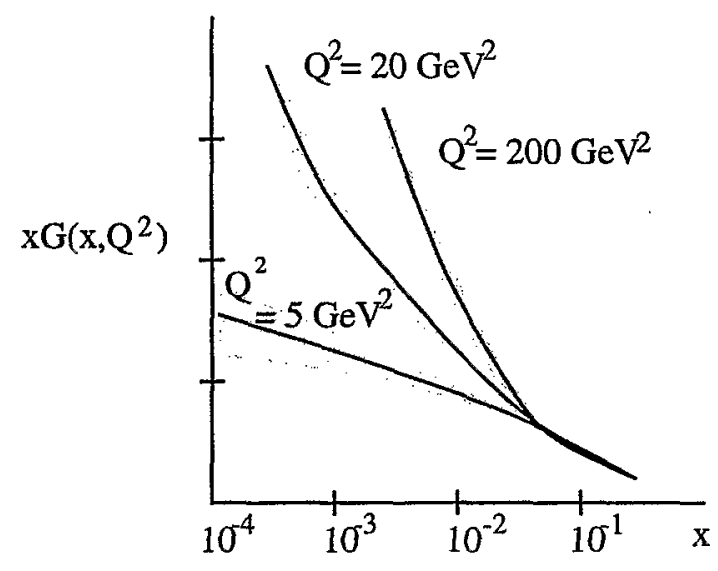

Figure 8: The number of gluons in a proton per unit rapidity at various rapidities and $Q^{2}$ resolutions.

Note that the gluon density rises rapidly at small $x$ in Fig. 8 . This is the so called small $\mathrm{x}$ problem. It means that if we view the proton head on at increasing energies, the low momentum gluon density grows. This is shown in Fig. 9.

As the density of gluons per unit area, per unit rapidity increases, the typical transverse separation of the gluons decreases. This means that the matter which controls high energy strong interactions is very dense, and it means that the QCD interaction strength, which is usually parameterized by the dimensionless scale $\alpha_{S}$ becomes small. The phase space density of these gluons, $\rho \sim 1 / \pi R^{2} d N / d^{2} p_{T}$ can become at most $1 / \alpha_{s}$ since once this density is reached gluon interactions are important. This is characteristic of bose condensation phenomena which are generated by an instability proportional to 


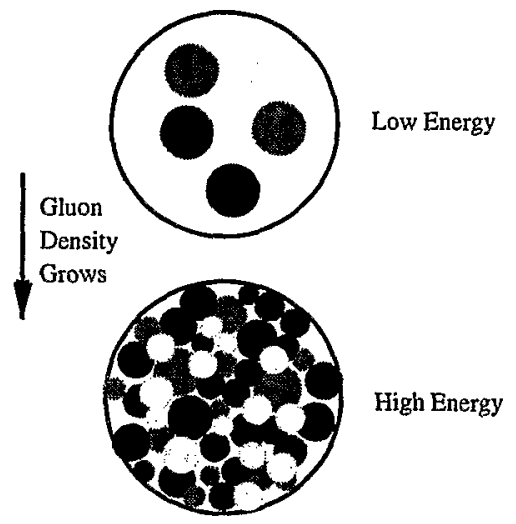

Figure 9: The increasing density of wee partons as the energy increases.

the density $\rho$ and is compensated by interactions proprotional to $\alpha_{S} \rho^{2}$, which become of the same order of magnitude when $\rho \sim 1 / \alpha_{s}$ Thus the matter is a Color Condensate.

The glassy nature of the condensate arise because the fields associated with the condensate are generated by constituents of the proton at higher momentum. These higher momentum constituents have their times scales Lorentz time dilated relative to those which would be meaured in their rest frame. Therefore the fields associated with the low momentum constituents also evolve on this long time scale. The low momentum constituents are therefore glassy: their time evolution scale is unnaturally long compared to their natural time scale. Hence the name Color Glass Condensate.

There is also a typical scale associated with the Color Glass Condensate: the saturation momentum. This is the typical momentum scale where the phase space density of gluons becomes $\rho<<1 / \alpha_{S}$.

At very high momentum, the fields associated with the Color Glass Condensate can be treated as classical fields, like the fields of electricity and magnetism. Since they arise from fast moving partons, they are plane polarized perpendicular to the direction of motion of the hadron. They are also random in two dimensions. This is shown in Fig. 10.

\subsubsection{Why is the Color Glass Condensate Important?}

The Color Glass Condensate is the universal form of matter from which all high energy hadrons are made. Like nuclei and electrons compose atoms, and 


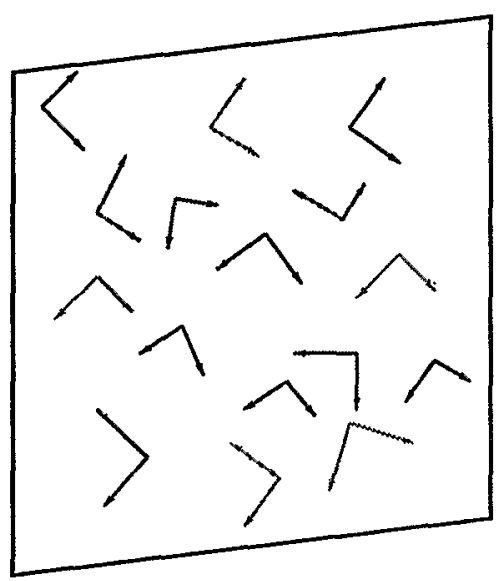

Figure 10: The Color Glass Condensate as a high density of random gluon fields on a two dimensional sheet travelling near the speed of light.

nucleons and protons compose nuclear matter, the Color Glass Condensate is the fundamental matter of which high energy hadrons are all composed.

The Color Glass Condensate has the potential to allow for a first principles description of the gross or typical properties of matter at high energies. For example, the total cross section at high energies for proton-proton scattering, as shown in Fig. 11 has a simple form but for over 40 years has resisted simple explanation. (It has perhaps been recently understood in terms of the Color Glass Condensate or Saturation ideas.)

The total hadronic cross section:

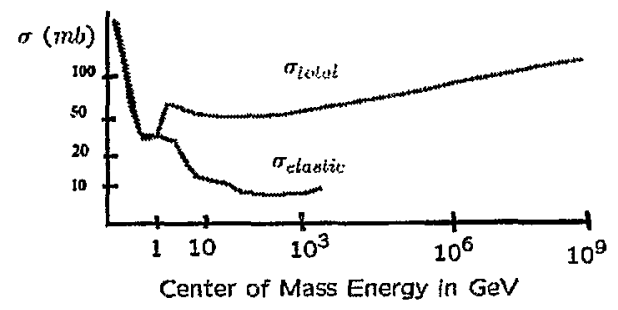

Figure 11: The total cross section for high energy proton-proton interactions.

The Color Glass Condensate form the matter in the quantum mechanical state which descirbes a nucleus. In the earliest stages of a nucleus-nucleus collisions, the matter must not be changed much from these quantum mechanical 
states. The Color Glass Condensate therefore provides the initial conditions for the Quark Gluon plasma to form in these collsions. A space-time picture of nucleus nucleus collisions is shown in Fig. 12. At very early times, the Color Glass Condensate evolves into a distribution of gluons. Later these gluons thermalize and may eventually form a Quark Gluon Plasma. At even later times, a mixed phase of plasma and hadronic gas may form.

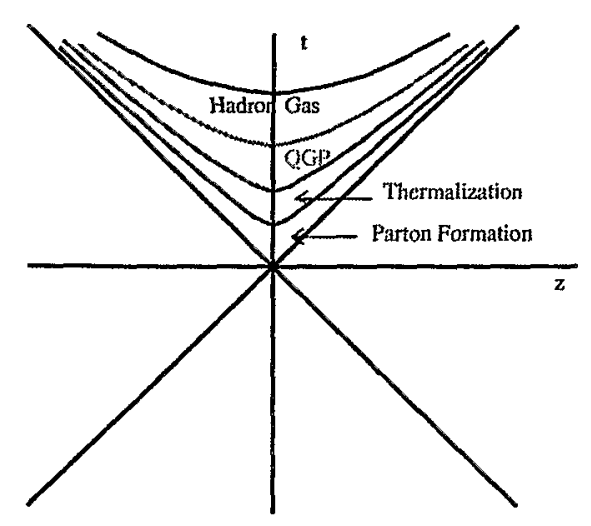

Figure 12: A space-time diagram for the evolution of matter produced in heavy ion collisions.

\section{Acknowledgements}

This manuscript has been authorized under Contract No. DE-AC02-98H10886 withthe U. S. Department of Energy.

[1] For a summary of recent results see the excellent review lectures by J. P. Blaizot, M. Gyulassy, and F. Karsch, , Lectures at the 40'th Internationale Universitatswochen fuer Theoretische Physik, Dense Matter, Schladming, Styria, Austria, 3-10 March 2001, hep-lat/0106019.

[2] For a summary of recent results see the review lectures by L. McLerran at the 40'th Internationale Universitatswochen fuer Theoretische Physik, Dense Matter, Schladming, Styria, Austria, 3-10 March 2001, heplat/0106019. 